05,13

\title{
Магнон-фононное взаимодействие в переходном слое эпитаксиальной пленки ЖИГ
}

\author{
(C) В.В. Тихонов, В.А. Губанов, А.В. Садовников \\ Саратовский государственный университет им. Н.Г. Чернышевского, \\ Саратов, Россия \\ E-mail: tvlad4@yandex.ru
}

Поступила в Редакцию 9 апреля 2021 г.

В окончательной редакции 9 апреля 2021 г.

Принята к публикации 19 апреля 2021 г.

Показано, что в переходном слое эпитаксиальной пленки ЖИГ возникают эффекты коллинеарного магнитоупругого взаимодействия обменных спиновых (ОСB) и акустических волн $(\mathrm{AB})$. Наиболее интенсивное взаимодействие происходит на частотах фазового синхронизма ОСВ и АВ. При этом появляются эффекты гибридизации и преобразования энергии $\mathrm{OCB} \rightarrow \mathrm{AB}$. Выполнение условия синхронизма обеспечивается трансформацией длин ОСВ в пределах толщины переходного слоя. Вдали от точек синхронизма связь ослабляется, волны распространяются независимо друг от друга. При этом акустическая волна может беспрепятственно излучаться вглубь немагнитной подложки пленки железоиттриевого граната. В перспективе это может быть полезным для обеспечения фононной связи спин-волновых возбуждений в пленках, расположенных на противоположных сторонах немагнитной подложки.

Ключевые слова: спиновые волны, магноника, фотоника, эпитаксиальные пленки.

DOI: 10.21883/FTT.2021.09.51310.32H

\section{1. Введение}

Дальнейшее развитие магнонной спинтроники стимулирует поиск новых механизмов транспорта спинового момента [1]. В частности исследуются возможности переноса спина когерентными фононами в композитных структурах, состоящих из ферромагнитных и акустических слоев [2,3]. Такие структуры получили название магнитоакустические метаматериалы [4]. К их числу относятся пленочные структуры железоиттриевого граната (ЖИГ), выращенные на подложках гадолиний-галлиевого граната (ГГГ). Структуры ЖИГ-ГГГ имеют ряд существенных преимуществ: ЖИГ обладает наименьшими магнитными потерями [5,6]; монокристаллы ЖИГ и ГГГ являются превосходными звукопроводами [7-9]; акустические импедансы в ЖИГ и ГГГ практически совпадают [10].

Магнитострикционные свойства ЖИГ обусловливали возможность гибридизации спиновых волн (магнонов) с акустическими волнами (фононами). Однако для наблюдения эффектов гибридизации необходимо было обеспечить условие фазового синхронизма [11-14]. Иными словами требовалось возбуждение обменных спиновых волн (ОСВ) сравнимых по длине волны с акустическими волнами (AB). Первоначально проблема возбуждения коротковолновых ОСВ решалась за счет уменьшения длины электромагнитной волны в неоднородном магнитном поле. Неоднородность внутреннего поля феррита обеспечивалась полями размагничивания на торцевых поверхностях массивных образцов цилиндрической формы $[15,16]$. Эффекты гибридизации и, соответственно, преобразования ОСВ $\rightarrow \mathrm{AB}$ возникали в окрестности точек пересечения парциальных законов дисперсии ОСВ и АВ. При этом в массивном образце возбуждались высокодобротные акустические резонансы.

Впоследствии аналогичные эффекты были обнаружены в пленках ЖИГ $[17,18]$. Отличие состояло в том, что локальная неоднородность внутреннего поля создавалась ионной имплантацией поверхностного слоя пленки ЖИГ. При этом возбуждались обменные спиновые и акустические волны, которые распространялись в поперечном направлении пленки. В импульсном режиме их можно было наблюдать в виде двух серий отраженных эхоимпульсов. Импульсы ОСВ отражались от противоположной поверхности пленки, а импульсы АВ излучались вглубь подложки ГГГ и отражались от ее противоположной поверхности. Аналогичные эффекты были обнаружены в специально изготовленных пленках ЖИГ с неоднородной намагниченностью насыщения по всей толщине пленки [19]. В недавних работах [20,21] было обнаружено эффективное возбуждения коротковолновых ОСВ в серийных образцах пленок ЖИГ. Волны возбуждались в тонком переходном слое на внутренней поверхности пленки ЖИГ. Их также можно было наблюдать либо в виде отраженных эхоимпульсов OCB, либо в виде стоячих волн на дискретных частотах спин-волновых резонансов.

Существование переходного слоя в пленке ЖИГ было обусловлено диффузией немагнитных ионов $\mathrm{Gd}^{3+}$, $\mathrm{Ga}^{3+}$ подложки ГГГ $\left(\mathrm{Gd}_{3} \mathrm{Ga}_{5} \mathrm{O}_{12}\right)$, замещающих магнитные ионы $\mathrm{Y}^{3+}, \mathrm{Fe}^{3+}$ пленки ЖИГ $\left(\mathrm{Y}_{3} \mathrm{Fe}_{5} \mathrm{O}_{12}\right)$ [22-24]. Это одинаково касалось пленок ЖИГ, выращенных 
методом жидкофазной эпитаксии [22,23] и методом ионно-лучевого распыления [24]. Было установлено, что в пленках ЖИГ, выращенных методом жидкофазной эпитаксии, толщина переходного слоя может составлять $0.2-0.5 \mu \mathrm{m}[23]$.

В настоящей работе исследовалась возможность гибридизации обменных спиновых и акустических волн в переходном слое пленки ЖИГ. Были сделаны оценки интенсивности возбуждения акустических волн при заданной интенсивности обменных спиновых волн.

\section{2. Структура и численное исследование}

Геометрия задачи представлена на вставке рис. 1, $a$. Рассматривалась двухслойная структура ЖИГ-ГГГ, на внутренней границе которой имелся тонкий переходный слой. Распределение намагниченности в переходном слое описывалось функцией $M(z)=M_{0}[1-\exp (-\sigma z)]$, где $M_{0}=140 \mathrm{G}$ - намагниченность чистого ЖИГ, $\sigma-$ параметр распределения намагниченности по толщине пленки. В дальнейших расчетах использовалось значение параметра $\sigma=82093 \mathrm{~cm}^{-1}$, полученное в работах [20,21]. Намагничивающее поле $\mathbf{H}_{0} \| \mathbf{z}$ ориентировалось по нормали к поверхности пленки, как показано на вставке рис. $1, a$.

Решалась задача взаимодействия обменных спиновых и сдвиговых упругих волн, распространяющихся в направлении волнового вектора $\mathbf{k}\left\|\mathbf{H}_{0}\right\| \mathbf{M}_{0}$. Решения искались в виде плоских волн циркулярной поляризации $m_{ \pm} \exp [i(\omega t-k z)], u_{ \pm} \exp [i(\omega t-k z)]$, где $m_{ \pm}=m_{x} \pm i m_{y}$ и $u_{ \pm}=u_{x} \pm i u_{y}-$ амплитуды прецессии вектора спонтанной намагниченности $\mathbf{m}$ и вектора упругого смещения $\mathbf{u}, k-$ волновое число связанных волн, $\omega=2 \pi f-$ круговая частота. Для простоты изложения затухание волн и кристаллографическая анизотропия пленки ЖИГ не учитывались.

Совместно решались уравнение Ландау-Лифшица, записанное с учетом неоднородного обмена, и уравнение движения упругой среды. С учетом сделанных ограничений эти уравнения приводились к виду $[25,26]$

$$
\begin{gathered}
{\left[\omega \mp\left(\omega_{H}+\eta k^{2}\right)\right] m_{ \pm}=-i k \gamma B_{2} u_{ \pm},} \\
\left(\omega^{2}-v_{\perp}^{2} k^{2}\right) u_{ \pm}=i k \frac{B_{2}}{\rho M} m_{ \pm} .
\end{gathered}
$$

где $\omega_{H}=\gamma\left[H_{0}-4 \pi M(z)\right], \gamma=1.76 \cdot 10^{7} \mathrm{Oe}^{-1} \mathrm{~s}^{-1}-$ гиромагнитное отношение, $\eta=8.62 \cdot 10^{6} \mathrm{~cm}^{2} \mathrm{~s}^{-1}$ - постоянная неоднородного обмена, $B_{2}=6.96 \cdot 10^{6} \mathrm{erg} / \mathrm{cm}^{3}-$ релятивистская магнитоупругая постоянная, $v_{\perp} \approx$ $\approx 3.84 \cdot 10^{6} \mathrm{erg} / \mathrm{cm}^{3}-$ скорость сдвиговых упругих волн, $\rho \approx 5.17 \mathrm{~g} / \mathrm{cm}^{3}$ - плотность ЖИГ. Следует отметить, что замещение магнитных ионов $\mathrm{Fe}^{3+}$ немагнитными ионами $\mathrm{Ga}^{3+}$ в переходной области ЖИГ/ГГГ приведет к изменению не только намагниченности пленки, но и постоянных $\eta$ и $B_{2}$. Однако в тех случаях, когда замещение ионов $\mathrm{Fe}^{3+}$ ионами $\mathrm{Ga}^{3+}$ в октаэдрической подрешетке оказывается выше, чем в тетраэдрической, величина намагниченности снижается быстрее, чем $\eta$ и $B_{2}$ [27-29]. Далее мы будем считать, что выполняется именно такой случай и значения $\eta$ и $B_{2}$ будем считать постоянными.

В дальнейшем нас интересовало наиболее интенсивное взаимодействие правополяризованных волн. Для этого случая связанные уравнения (1a), (1b) можно было представить в более удобном виде

$$
\begin{aligned}
& \left(k_{m 0}^{2}-k^{2}\right) m_{+}=-i k \frac{\gamma B_{2}}{\eta} u_{+}, \\
& \left(k_{s 0}^{2}-k^{2}\right) u_{+}=i k \frac{B_{2}}{\rho v_{\perp}^{2} M} m_{+},
\end{aligned}
$$

где $k_{m 0}=\sqrt{\left(\omega-\omega_{H}\right) / \eta}$ и $k_{s 0}=\omega / v_{\perp}$ - волновые числа несвязанных ОСВ и АВ. Из уравнений $(2 \mathrm{a})$ и $(2 \mathrm{~b})$ нетрудно было получить дисперсионное уравнение гибридных магнитоакустических волн

$$
\left(k_{m 0}^{2}-k^{2}\right)\left(k_{s 0}^{2}-k^{2}\right)=\frac{\xi}{\alpha} k^{2},
$$

где $\xi=B_{2}^{2} /\left(\rho v_{\perp}^{2} M^{2}\right)-$ безразмерный параметр магнитоупругой связи. Также можно было получить отношение квадратов амплитуд гибридизованных волн

$$
\frac{u_{+}^{2}}{m_{+}^{2}}=\frac{\alpha}{\rho v_{\perp}^{2}} \frac{\left(k_{m 0}^{2}-k_{m}^{2}\right) k_{s}}{\left(k_{s 0}^{2}-k_{s}^{2}\right) k_{m}} .
$$

Уравнение (3) имело простое аналитическое решение относительно волнового числа

$$
\begin{aligned}
& k_{m, s}= \\
& =\sqrt{\frac{1}{2}\left(k_{m 0}^{2}+k_{s 0}^{2}+\frac{\xi}{\alpha}\right) \pm \sqrt{\frac{1}{4}\left(k_{m 0}^{2}+k_{s 0}^{2}+\frac{\xi}{\alpha}\right)^{2}-k_{m 0}^{2} k_{s 0}^{2}}},
\end{aligned}
$$

где знак $(+)$ соответствовал волновому числу магнитной составляющей гибридной волны $k_{m}$, а знак (-) волновому числу упругой составляющей $k_{s}$.

Выражение (4) само по себе не имело смысла, поскольку представляло собой отношение величин разной размерности. Тем не менее, оно было необходимо для расчета отношения величин потоков мощности $\chi=\prod_{s} / \prod_{m}, \quad$ переносимых упругой $\prod_{s}=(1 / 2) \rho \omega^{2} u_{+}^{2} v_{s g}$ и магнитной составляющей $\prod_{m}=(1 / 2)\left[\left(H_{0}-4 \pi M\right) / M\right] m_{+}^{2} v_{m g} \quad$ гибридной волны [26]. Здесь $v_{s g}=\left(\partial k_{s} / \partial \omega\right)^{-1}$ и $v_{m g}=\left(\partial k_{m} / \partial \omega\right)^{-1}-$ групповые скорости упругой и магнитной составляющей. С учетом (4) нетрудно было получить формулу расчета отношения потоков мощности в виде

$$
\chi=\frac{k_{s 0}^{2} \eta}{\omega_{H}} \frac{\left(k_{m 0}^{2}-k_{m}^{2}\right) k_{s}}{\left(k_{s 0}^{2}-k_{s}^{2}\right) k_{m}} \frac{v_{s g}}{v_{m g}} .
$$



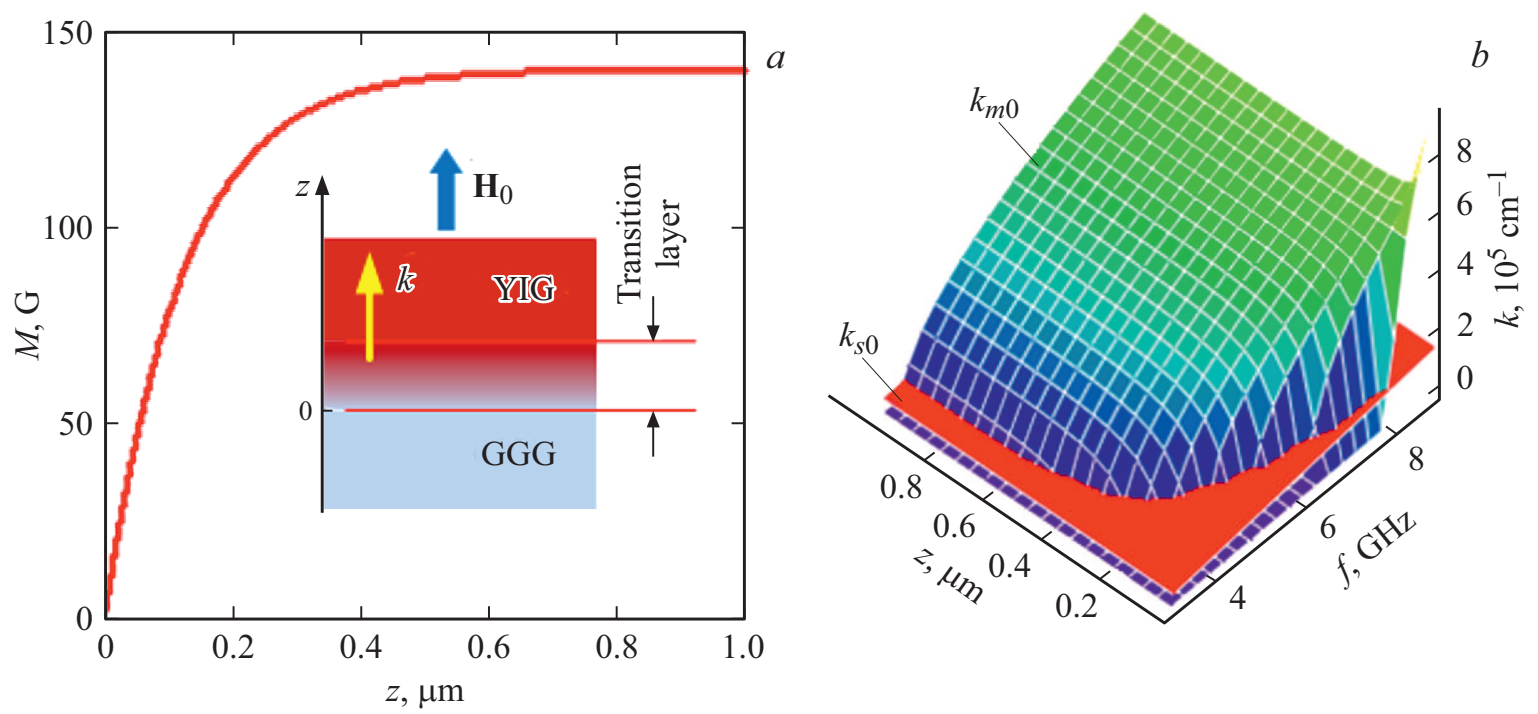

Рис. 1. Результаты предварительных расчетов: (a) распределение намагниченности в переходном слое пленки ЖИГ (на вставке: геометрия задачи); (b) парциальные законы дисперсии обменных спиновых волн $k_{m 0}(f, z)$ и акустических волн $k_{s 0}(f)$.
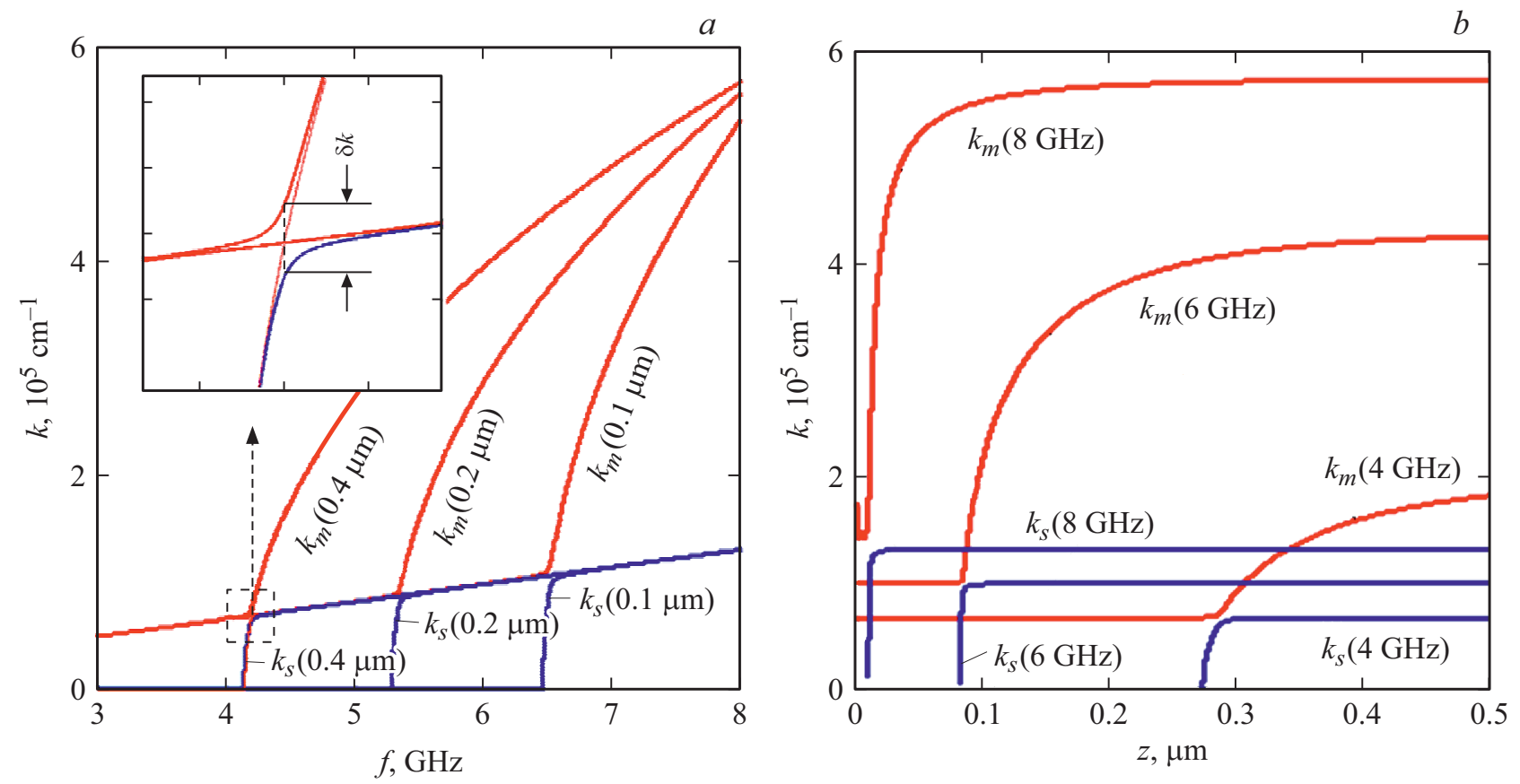

Рис. 2. Дисперсия магнитоакустических волн: $(a)$ магнитная составляющая гибридной волны $k_{m}(f, z)$, рассчитанная при фиксированных значениях координат $z=0.1,0.2,04 \mu \mathrm{m} ;(b)$ акустическая составляющая гибридной волны $k_{s}(f, z)$, рассчитанная при фиксированных значениях частоты $f=4,6,8 \mathrm{GHz}$.

Ниже приведены результаты численных расчетов, которые проводились при фиксированном значении намагничивающего поля $H_{0}=3000$ Ое. Расчетная зависимость распределения намагниченности $M(z)$ без учета перемешивания ионов $\mathrm{Gd}^{3+}, \mathrm{Ga}^{3+}, \mathrm{Y}^{3+}$ и $\mathrm{Fe}^{3+}$ представлена на графике рис. $1, a$. Из графика следует, что толщина переходного слоя составляла приблизительно $0.5 \mu \mathrm{m}$. В этой области исследовались условия гибридизации связанных волн. С этой целью строились $3 d$-графики парциальных законов дисперсии ОСВ и АВ, которые с учетом координатной зависимости $M(z)$ имели вид

$$
\begin{gathered}
k_{m 0}(f, z)=\sqrt{\frac{2 \pi f-\gamma\left[H_{0}-4 \pi M(z)\right]}{\gamma \alpha M(z)},} \\
k_{s 0}(f)=\frac{2 \pi f}{v_{\perp}} .
\end{gathered}
$$

Условие синхронизма ОСВ и $\mathrm{AB}$ выполнялось в точках, расположенных на линии пересечения дисперси- 

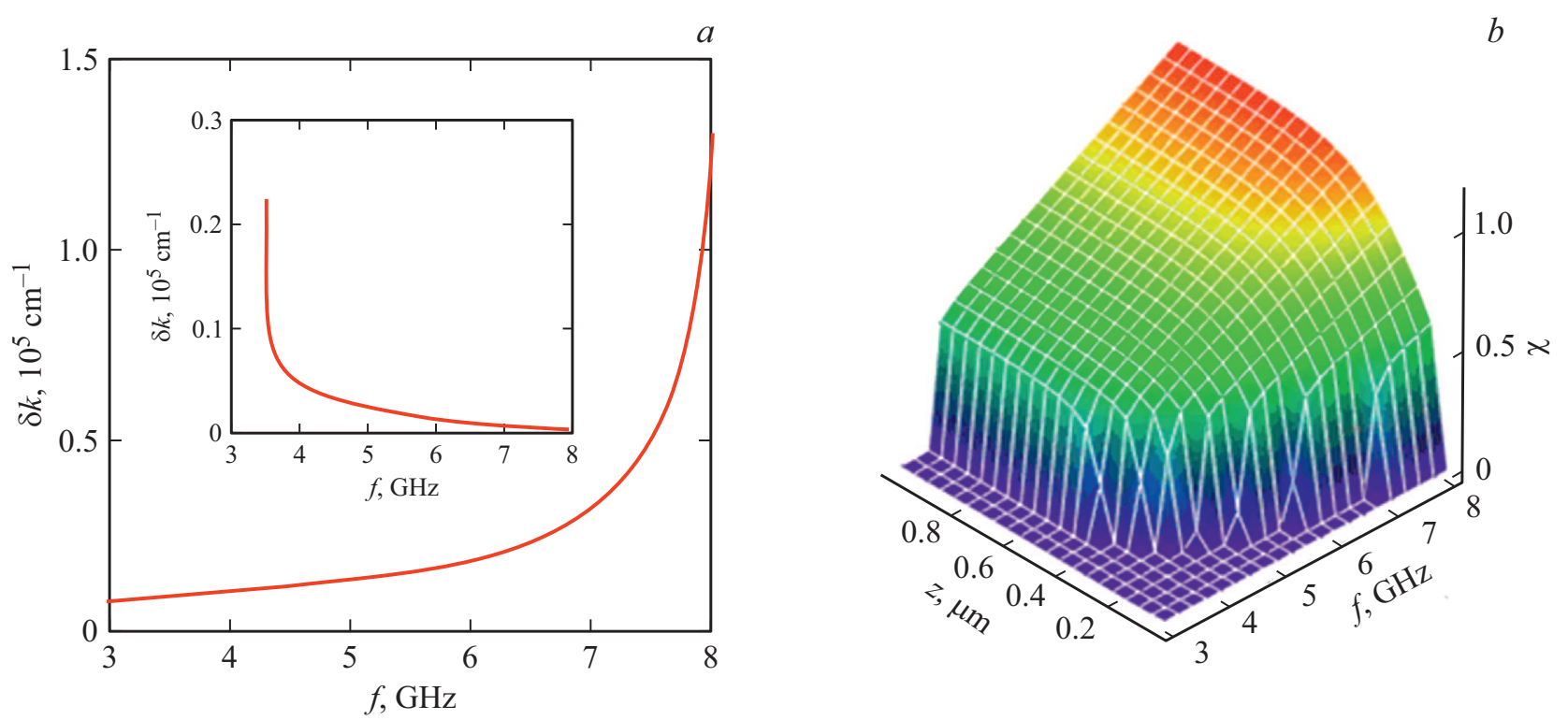

Рис. 3. Результаты расчета параметров связи $(a)$ и интенсивности преобразования $\mathrm{OCB} \rightarrow \mathrm{AB}(b)$ в переходном слое ЖИГ-ГГГ.

онных поверхностей $k_{m 0}(f, z)$ и $k_{s 0}(f)$, как показано на рис. $1, b$.

На графике рис. $1, b$ хорошо видно, что в пределах толщины переходного слоя существует широкая полоса частот, в которой выполняются условия гибридизации OCB и AB, причем координаты точек гибридизации $z_{0}$ зависят от частоты возбуждения. Используя условие синхронизма $k_{m 0}\left(f, z_{0}\right)=k_{s 0}(f)$, нетрудно было получить частотную зависимость $z_{0}(f)$ в следующем виде

$$
\begin{aligned}
& z_{0}(f)=\frac{1}{\sigma} \\
& \times \ln \left[\frac{(2 \pi f)^{2} / \nu_{\perp}^{2}-4 \pi / \alpha}{(2 \pi f)^{2} / v_{\perp}^{2}-\left[2 \pi f-\gamma\left(H_{0}-4 \pi M_{0}\right)\right] / \gamma \alpha M_{0}}\right] .
\end{aligned}
$$

График зависимости $z_{0}(f)$ представлен на вставке рис. $3, a$.

На рис. 2 представлены дисперсионные зависимости магнитной $k_{m}(f, z)$ и упругой составляющей гибридной волны $k_{s}(f, z)$, рассчитанные при фиксированных значениях координат (рис. 2,a) и при фиксированных значениях частоты (рис. 2,b). В обоих случаях вблизи точек синхронизма $z_{0}(f)$ возникала раздвижка дисперсионных ветвей, как показано на вставке рис. 2, $a$. При этом ширина щели $\delta k(f)=k_{m}\left(f, z_{0}(f)\right)-k_{s}\left(f, z_{0}(f)\right)$ существенно зависела от частоты возбуждения.

График частотной зависимости ширины щели $\delta k(f)$ представлен на рис. 3,a. Видно, что с ростом частоты ширина щели возрастала, достигая максимума вблизи верхней границы частот гибридизации. Для сравнения на вставке рис. 3, $a$ представлен график частотной зависимости координаты точек синхронизации $z_{0}(f)$. На рис. $3, b$ представлен $3 d$-график отношения потоков мощности, переносимых упругой и магнитной составляющей гибридной волны $\chi(f, z)$. Видно, что вблизи верхней границы частот гибридизации интенсивность возбуждения упругой составляющей может превышать интенсивность магнитной составляющей.

\section{3. Заключение}

На основании проведенных исследований, было показано, что неоднородность пленки ЖИГ играет важную роль в процессах преобразования типа волн. В настоящей работе рассматривались эффекты преобразования $\mathrm{OCB} \rightarrow \mathrm{AB}$, которые возникали за счет коллинеарного магнитоупругого взаимодействия. Наиболее сильное взаимодействие возникало на частотах фазового синхронизма (гибридизации) ОСВ и АВ. Условия синхронизма обеспечивались трансформацией длин ОСВ в переходном слое эпитаксиальной пленки ЖИГ. Вдали от точек синхронизма магнитоупругая связь значительно ослаблялась, что обуславливало независимое распространение обменных спиновых и акустических волн. При этом акустическая волна могла беспрепятственно излучаться в подложку ГГГ. Последнее обстоятельство обеспечивает возможность фононной связи спин-волновых возбуждений в пленках ЖИГ, расположенных на противоположных сторонах подложки. Это, в свою очередь, открывает дополнительные возможности развития нового направления $3 d$-магноники.

\section{Финансирование работы}

Работа выполнена при поддержке Минобрнауки России в рамках выполнения государственного задания (проект № FSRR-2020-0005). 


\section{Конфликт интересов}

Авторы заявляют, что у них нет конфликта интересов.

\section{Список литературы}

[1] A. Hirohata, K. Yamada, Y. Nakatani, I.-L. Prejbeanu, B. Dieny, P. Pirro, B. Hillebrands. J. Magn. Magn. Mater. 509, 166711 (2020).

[2] K. Oyanagi, S. Takahashi, L.J. Cornelissen, J. Shan, S. Daimon, T. Kikkawa, G.E.W. Bauer, B.J. vanWees, E. Saitoh. Nature Commun. 10, 4740 (2019).

[3] J. Holanda, D.S. Maior, A. Azevedo, S.M. Rezende. Nature Phys. 14, 500 (2018).

[4] Ю.В. Гуляев, С.В. Тарасенко, В.Г. Шавров. УФН 181, 595 (2011). [Yu.V. Gulyaev, S.V. Tarasenko, V.G. Shavrov. Phys. Uspekhi 54, 6, 573 (2011).].

[5] E.G. Spencer, R.C. LeCraw, A.M. Clogston. Phys. Rev. Lett. 3, 32 (1959).

[6] V. Cherepanov, I. Kolokolov, V. L'vov. Phys. Rep. 229, 81 (1993).

[7] R.C. Le Craw, E.G. Spencer, E.I. Gordon. Phys. Rev. Lett. 6, 620 (1961).

[8] E.G. Spencer, R.T. Denton, R.P. Chambers. Phys. Rev. 125, 1950 (1962).

[9] E.G. Spencer, R.T. Denton, T.B. Bateman, W. B. Snow, L.G.V. Uitert. J. Appl. Phys. 34, 3059 (1963).

[10] N. Polzikova, S. Alekseev, V. Luzanov, A. Raevskiy. J. Magn. Magn. Mater. 479, 38 (2019).

[11] C. Kittel. Phys. Rev. 110, 836 (1958).

[12] H. Bömmel, K. Dransfeld. Phys. Rev. Lett. 3, 83 (1959).

[13] R. Damon, H. van de Vaart. Proc. IEEE 53, 348 (1965).

[14] M. Seavey. Proc. IEEE 53, 1387 (1965).

[15] J.R. Eshbach. J. Appl. Phys. 34, 1298 (1963).

[16] E. Schlömann, R.I. Joseph. J. Appl. Phys. 35, 2382 (1964).

[17] Ю.В. Гуляев, П.Е. Зильберман, Е.С. Санников, В.В. Тихонов, А.В. Толкачев. Письма в ЖТФ 14, 884 (1988).

[18] В.В. Тихонов, А.В. Толкачев. ФТТ 36, 1, 185 (1994).

[19] П.Е. Зильберман, А.Г. Темирязев, М.П. Тихомирова. ЖЭТФ 108, 281 (1995).

[20] V.V. Tikhonov, A.N. Litvinenko. Appl. Phys. Lett. 115, 072410 (2019).

[21] V.V. Tikhonov, A.N. Litvinenko. J. Magn. Magn. Mater. 515, 167241 (2020).

[22] M. Shone. Circuits Syst. Signal Proc. 4, 89 (1985).

[23] С.И. Ющук. ЖТФ 69, 62 (1999).

[24] M.-B. Park, N.-H. Cho. J. Magn. Magn. Mater. 231, 253 (2001).

[25] Физическая акустика / Под ред. У. Мэзона. Динамика решетки. Мир, М. (1968). Т. 3. 392 с.

[26] А.Г. Гуревич, Г.А.Мелков. Магнитные колебания и волны. Физматлит, М. (1994). 464 с.

[27] P. Hansen. J. Appl. Phys. 45, 8, 3638 (1974).

[28] С. Крупичка. Физика ферритов и родственных им магнитных окислов. Мир, М. (1976). Т. 1,2. 864 с.

[29] S. Geller, H.J. Williams, R.C. Sherwood, G.P. Espinosa. J. Appl. Phys. 36, 88 (1965).

Редактор Ю.Э. Китаев 